\title{
Presença da fisioterapia preventiva no contexto escolar: educação e prevenção em saúde
}

\author{
Preventive physiotherapy in school context: \\ health education and prevention
}

\author{
Kamila Mohammad Kamal Mansour, ${ }^{1}$ Patrícia Oliveira Roveda ${ }^{1}$ \\ 'Universidade de Santa Cruz do Sul (Unisc), Santa Cruz do Sul, RS, Brasil.
}

Recebido em: 23/07/2016 / Aceito em: 26/09/2016 / Publicado em: 18/10/2016

kamilamkmansour@gmail.com

\section{RESUMO}

Objetivo: este estudo tem por finalidade explicitar as ações da fisioterapia nas escolas, analisando alguns dos dados coletados durante uma das práticas da disciplina, em escola privada do município de Santa Cruz do Sul-RS. Método: estudo de caráter descritivo, foi composto por 206 estudantes, com idades entre 6 e 10 anos, de ambos os sexos, sendo alunos do $1^{\circ}$ ao $4^{\circ}$ ano do Ensino Fundamental, distribuídos em 11 turmas, de uma escola privada. A abordagem consistiu na "Educação Postural" no ambiente escolar e nas atividades de vida diárias, aplicação de um questionário de múltipla escolha que englobava a avaliação do aspecto biopsicossocial do aluno. Foi feita a avaliação postural estática, assim como da postura de cada escolar frente ao mobiliário em sala de aula, através de análise observacional individual, sendo realizada a pesagem dos alunos, das mochilas e o ajuste das mesmas também foi realizada. Resultados: a amostra foi composta por 206 estudantes do $1^{\circ}$ ao $4^{\circ}$ ano do Ensino Fundamental. com idade entre seis e 10 anos, de ambos os sexos. Os achados provenientes do questionário nos forneceram informações acerca da realidade de vida de cada aluno avaliado. Em relação às avaliações posturais realizadas, foram evidenciados quatro casos de escoliose em " $\mathrm{C}$ " na coluna lombar e um em "S", já na coluna torácica foram encontrados sete casos de escoliose em " $\mathrm{C}$ ". $\mathrm{O}$ mobiliário se mostrou inadequado para a maioria dos estudantes e em relação ao peso das mochilas, 148 delas estavam acima dos $10 \%$ do peso corporal do aluno. Considerações finais: existe um vasto campo de atuação para a Fisioterapia Preventiva, dentre eles, a avaliação e intervenção na saúde na escola. As metodologias utilizadas na disciplina para esta prática possibilitaram o aprendizado e a [re] significação da prevenção na Fisioterapia, uma profissão fortemente enraizada na reabilitação.

Palavras-chave: Saúde Escolar; Fisioterapia; Promoção da Saúde; Educação em Saúde.

\section{ABSTRACT}

Objective: the aim of this study was to explicit the physiotherapy actions in schools, analyzing some of the data collected during one of the practical classes of the discipline in a private school set in Santa Cruz do Sul-RS. Method: descriptive study whose sample was composed by 206 students from 11 classes from elementary school, all of them from the same private school. The approach consisted of "Postural education" at school and in daily life activities, application of a multiple choice questionnaire that assessed the biopsychosocial aspects of the schoolchild. We also assessed the static posture and the posture of each student using the furniture in the classroom through individual observational analysis. The weighing of students, backpacks and their adjusting was also performed. Results: the sample consisted of 206 students, aged six to 10 years, of both genders, from 1st to 4th grade of elementary school. The findings from the questionnaire provided us with information about the quality of life of each student assessed. In the postural evaluations we found four cases of scoliosis in " $C$ " in the lumbar spine and one in " $S$ ", as in the thoracic spine we have found seven cases of scoliosis in " $C$ ". The furnishings showed inadequate for most students and regarding the weight of backpacks, 148 of them were above $10 \%$ of the body weight of the student. Closing remarks: there is a vast field of action for Preventive Physiotherapy, including the assessment 
and intervention in health at school. The methodologies used in practice of the discipline enabled the learning and the [re] significance of prevention in physiotherapy, a profession deeply rooted in rehabilitation.

Keywords: School Health; Physiotherapy; Health Promotion; Health Education.

\section{INTRODUCÃ̃O}

A escola é um importante local para o encontro entre saúde e educação, proporcionando diversas possibilidades de ações de cuidado e prevenção como: ações de diagnóstico clínico e/ou social, estratégias de triagem e/ou encaminhamento aos serviços de saúde, especializados ou de atenção básica, além de atividades de educação em saúde e promoção da saúde que podem ser amplamente realizadas. ${ }^{1}$

Os primeiros anos de vida escolar são fundamentais para agregar e automatizar bons hábitos, dentre eles os posturais, sendo de grande importância a estimulação e execução adequadas das atividades de vida diária, visando a promoção da saúde cinético-funcional dos escolares. ${ }^{2} \mathrm{~A}$ atuação em todos os níveis de atenção em saúde, como em programas de prevenção, promoção, manutenção, proteção e recuperação da saúde está entre as competências e habilidades na formação do Fisioterapeuta. ${ }^{3}$

A fisioterapia, em ambiente escolar, vem atuando de maneira preventiva, através da educação em saúde, com o foco nos hábitos posturais. Os programas propostos para tal população demonstram estar repercutindo positivamente nas práticas de vida diária dos mesmos. ${ }^{4-6}$

A disciplina de Fisioterapia Preventiva na Comunidade II do Curso de Fisioterapia na Universidade de Santa Cruz do Sul tem como objetivo despertar e estimular a importância da prevenção, proporcionando ao discente, uma atuação fisioterápica preventiva nos diferentes locais da comunidade em que está inserido, capacitando o aluno a identificação e aplicação de métodos fisioterápicos preventivos mais adequados na comunidade.

Este estudo objetivou explicitar as ações da fisioterapia nas escolas, analisando alguns dos dados coletados, durante uma das práticas da disciplina em uma escola privada do município de Santa Cruz do Sul-RS.

\section{MÉTODO}

O estudo de caráter descritivo, foi composto por 206 estudantes, com idades entre 6 e 10 anos, de ambos os sexos, sendo alunos do $1^{\circ}$ ao $4^{\circ}$ ano do Ensino Fundamental, distribuídos em 11 turmas, de uma escola privada. Tal atividade consistiu em atividade integrante da disciplina de Fisioterapia Preventiva na Comunidade II.

Através das aulas práticas da disciplina de Fisioterapia Preventiva na Comunidade II, acadêmicos e docente estiveram em escolas públicas e privadas da cidade de Santa Cruz do Sul, sendo este um estudo descritivo, cuja amostra foi composta por escolares distribuídos em 11 turmas do Ensino Fundamental, todos de uma mesma escola privada. Todos os alunos que compareceram à aula foram avaliados, não havendo critérios específicos de inclusão ou exclusão, pois a ida à escola foi de caráter investigativo e preventivo. Todos os procedimentos foram realizados no mesmo dia.

A primeira ação, diretamente com os escolares, foi o uso de diferentes estratégias com o intuito de promover a "Educação Postural" no ambiente escolar e nas atividades de vida diárias. Dentre as estratégias utilizadas destacamos: a palestra interativa, uso de jogos de fixação/memória, elaboração de cartazes, distribuição de folders, cruzadinhas, jogo de tabuleiro, entre outras. Em seguida, foi aplicado um questionário de múltipla escolha que englobou a avaliação do aspecto biopsicossocial do aluno. Dando continuidade à intervenção foi realizada a avaliação postural estática, a postura de cada escolar frente ao mobiliário em sala de aula, através de análise observacional individual bem como foi realizada a pesagem dos alunos, das mochilas e o ajuste das mesmas.

\section{RESULTADOS E DISCUSSÃO}

Observou-se, de modo bastante positivo, a interação e interesse dos escolares, durante a realização das diferentes estratégias de Educação Postural, cujo relato de experiência evidenciou a importância desta ação.

Borba e Melo ${ }^{7}$ propõem trabalhar com os escolares de forma lúdica, por meio de jogos e brincadeiras como forma de estimular a imaginação e o raciocínio, possibilitando o desenvolvimento da curiosidade, criatividade e autonomia. Estas estratégias promovem o desenvolvimento intelectual, agindo diretamente na psicomotricidade e no raciocínio da criança, garantindo a construção e aquisição de conhecimentos. Já Cardoso et al. ${ }^{8}$ comparou a efetividade de histórias em quadrinhos e do teatro de fantoches na aprendizagem de hábitos posturais em escolares. Na avaliação, após dois meses da intervenção educativa, constatou-se que as duas formas são efetivas para ensinar e fixar conceitos sobre os hábitos posturais corretos. Conforme explanado na metodologia deste trabalho, muitas destas estratégias/ações, citadas pelos autores, foram efetivadas na escola, pois através do brincar, compartilhamos o conhecimento teórico aos educandos.

O questionário biopsicossocial, construído pelos alunos da disciplina, foi respondido a partir dos alunos do $2^{\circ}$ ano, com auxílio dos acadêmicos da Fisioterapia, totalizando a análise de 150 estudantes, justificando a importância de se investigar aspectos biopsicossociais mencionamos Suls e Rothman ${ }^{9}$ que afirmam que é fundamental que o modelo biopsicossocial contemple os aspectos biológicos, psicológicos, sociais e culturais dos alunos. Outro estudo, proposto por Ferrari et al. ${ }^{10}$ através de um projeto de educação de adolescentes para a promoção da saúde e prevenção de doenças, utilizou o diagnóstico biopsicossocial em alunos de 14 a 19 anos, descobrindo-se que, dentre outros aspectos, estes alunos possuíam alimentação inadequada, baixa autoestima, sedentarismo e que utilizavam tabaco, álcool e outras drogas. Nosso questionário abrangeu os quatro aspectos supramencionados, com o propósito de identificar o cenário em que se encontram os educandos avaliados.

O questionário utilizado no presente estudo avaliou o deslocamento até a escola, em que 93,19\% chegam 
ao seu destino via ônibus/van/carro; quanto à alimentação $98,65 \%$ se alimentam antes de irem à escola; $46,97 \%$ dos estudantes dormem em torno de 9 a 10 horas; $51,33 \%$ dos alunos já tiveram alguma orientação postural; $34,4 \%$ já fizeram algum tratamento médico ou fisioterapêutico. Quanto à presença de dor ou desconforto físico, $62,41 \%$ assinalou a opção "às vezes", sendo a região da coluna lombar a mais mencionada. Os indivíduos apresentaram preferência para dormir em decúbito lateral. Na questão referente a gostar de ir para a escola, 97,35\% assinalaram que "sim"; sobre o tipo de atividade extracurricular mais praticada, os alunos responderam assinalando mais de uma opção, resultando nas seguintes respostas: 57 alunos (28\%) "computador/videogame/televisão", 85 alunos (41\%) "prática de esportes" e 46 (22\%) a opção "outros".

Santos, ${ }^{12}$ ao investigar a prevalência de dor na coluna e a associação desta com os hábitos posturais de vida diária em escolares do Ensino Fundamental II, na cidade de Campina Grande-PB constatou que a prevalência de dor na coluna foi de $49,5 \%$, sendo a região torácica a mais acometida $(19,4 \%)$, dados que corroboram, parcialmente, com o presente trabalho. Já, Lemos et al. ${ }^{11}$ avaliou a ocorrência de dor lombar e fatores associados em estudantes de 7 a 17 anos, e assim como realizado durante nossa prática, sua intervenção foi feita em uma escola privada e a avaliação da dor foi avaliada através de questionário.

Quanto às avaliações estáticas da postura, foram avaliados 82 escolares, tendo sido observado anteriorização de ombro em $46,34 \%$ dos casos e o valgo de joelho em 28,04\%. Espandar et al. ${ }^{13}$ em seu artigo de revisão observou que o joelho varo e o joelho valgo são deformidades angulares comuns durante a infância, mas geralmente trazem preocupações para os pais. Essas deformidades de natureza benigna representam variações normais do crescimento e desenvolvimento da criança, não necessitando de tratamento, apenas observação por parte dos profissionais da saúde.

Dentre os alunos avaliados foram encontrados quatro casos de escoliose em " $\mathrm{C}$ " na coluna lombar $\mathrm{e}$ um em "S", já na coluna torácica, foram encontrados sete casos de escoliose em " $\mathrm{C}$ ". Badaró et al. ${ }^{14}$ ressaltam que existe uma grande diferença na prevalência da escoliose devido à variabilidade dos critérios utilizados para o seu diagnóstico. Acredita-se que isso se deve ao fato de haver uma falta de critérios para diferenciar atitude escoliótica, escoliose postural e escoliose estrutural. Como a intervenção na escola foi pontual, não foi realizado estudo para diferenciar as curvaturas encontradas.

Sobre a avaliação do estudante no mobiliário, 37 deles não conseguiam manter a coluna alinhada ao encosto da cadeira, como também manter os ante pés apoiados no chão. A maioria deles (78,04\%) não conseguiam manter os pés com toda a planta apoiada no chão quando solicitado para sentarem corretamente, sendo fatores que predispõe a ocorrência de alterações posturais.

O uso contínuo de mesas e cadeiras inadequadas por escolares favorece a má postura, pela falta de apoio lombar e apoio para os pés. Mesas muito baixas ou cadeiras altas demais, levam a uma inclinação anterior da cabeça e do tronco para a execução das tarefas, au- mentando a pressão no disco intervertebral, enquanto que mesas muito altas ou cadeiras muito baixas levam a um deslocamento lateral dos braços, movimentando o centro de massa lateralmente. ${ }^{15}$

Tanto o mobiliário inadequado quanto à estatura do estudante e a má postura dos mesmos, mantida durante o turno escolar, são fatores geradores de dor, relação também encontrada neste estudo. Uma solicitação dos alunos entrevistados foi a aquisição de cadeiras estofadas, para oferecer maior conforto e a melhoria da altura das cadeiras, conforme estatura.

Meireles et al., ${ }^{16}$ ao observarem a influência de dois modelos diferentes de carteiras escolares na postura de alunos da rede pública da cidade de Cajazeiras, constatou que os dois modelos de assentos adotados pelas instituições têm sido comprometedores à saúde do estudante, pois foi alta a prevalência de alterações posturais frente ao mobiliário, mais de $90 \%$ dos escolares apresentou desvios.

Pouco mais da metade dos alunos (51,94\%) faz uso de mochila de duas alças; a maioria deles relata que apenas leva para a escola o material realmente necessário $(68,05 \%)$ e $47,08 \%$ relata que não considera sua mochila pesada. Mesmo com a falta de um valor de referência exato para o peso das mochilas escolares, a grande parte dos profissionais consente que elas não devem exceder $10 \%$ da massa corporal do aluno. ${ }^{17} \mathrm{De}$ um total de 206 mochilas pesadas, 148 delas estavam acima dos $10 \%$ recomendados, o que equivale a $71,84 \%$ da população escolar avaliada.

No momento da pesagem, todos os alunos foram orientados em relação ao modo correto de usar a sua mochila. O ideal é que as mochilas escolares possuam duas tiras largas e acolchoadas para os ombros, acolchoamento posterior e cinto abdominal, regulada para que fique na altura da cintura, terminando no início da região glútea. No caso das mochilas de rodinha, a alça do carrinho deve estar a uma altura apropriada ao tronco ereto ao puxá-la. A respeito da organização dos materiais dentro das mochilas, orientamos para que os materiais mais pesados devem ser colocados mais próximos das costas, concentrando os objetos mais pesados no centro das mesmas. ${ }^{18}$

\section{CONSIDERAÇÕES FINAIS}

Existe um vasto campo de atuação para a Fisioterapia Preventiva, dentre eles a avaliação e intervenção na saúde na escola tendo o presente estudo avaliado os escolares. Durante a prática, após transposto e compartilhado o conteúdo, prioritariamente, referente à Educação Postural de forma lúdica e de acordo com a faixa etária, produzindo um retorno positivo através da receptividade e curiosidade manifestada durante as atividades. De qualquer modo, ficaram evidentes os problemas encontrados no ambiente escolar, que podem trazer uma série de problemas de saúde. As metodologias utilizadas na disciplina para esta prática possibilitaram o aprendizado e a [re] significação da prevenção na Fisioterapia, uma profissão fortemente enraizada na reabilitação. 


\section{REFERÊNCIAS}

1. Casemiro JP, Fonseca ABC, Secco FVM. Promover saúde na escola: reflexões a partir de uma revisão sobre saúde escolar na América Latina. Ciênc Saúde coletiva 2014;19(3):829-40. doi: 10.1590/1413-81232014193.00442013

2. Noll M, Candotti CT, Vieira A. Escola postural: revisão sistemática dos programas desenvolvidos para escolares no Brasil. Movimento 2012;18(4):265-291.

3. Brasil. Diretrizes Curriculares Nacionais do Curso de Graduação em Fisioterapia [Internet]. Brasília: Conselho federal de educação; 19 fev. 2002. [acessado em 11 jul. 2016]. Disponível em: http://portal.mec.gov.br/component/ content/article? id =12991

4. Fernandes SMS, CasarottoRA, JOAOSMA. Efeitos desessões educativas no uso das mochilas escolares em estudantes do ensino fundamental I. Rev Bras Fisioter 2008;12(6):447-453. doi: 10.1590/S1413-35552008005000002.

5. Benini, J, Karolczak, APB. Benefícios de um programa de educação postural para alunos de uma escola municipal de Garibaldi, RS. Fisioterapia e Pesquisa 2010;17(4):346-351. doi: 10.1590/S1809-29502010000400012.

6. Vieira A, Treichel TL, Candotti CT, Noll M, Bartz PT. Efeitos de um Programa de Educação Postural para escolares do terceiro ano do Ensino Fundamental de uma escola estadual de Porto Alegre (RS). Fisioterapia e Pesquisa 2015;22(3):239 245. doi: 10.590/1809-2950/13228322032015.

7. Borba VR de Souza, Melo LS. O lúdico como possibilidade de intervenção no desenvolvimento biopsicossocial da criança no espaço escolar. Nucleus 2010;7(2):9-16. doi: 10.3738/ nucleus.v7i2.371.

8. Cardoso ARNR, Vasconcelos TB de, Josino JB, Arcanjo GN. Ensino de hábitos posturais em crianças: história em quadrinhos versus teatro de fantoches. Revista Brasileira em Promoção da Saúde 2014;27(3):319-326. doi: 10.5020/18061230.2014.p319.

9. Suls J, Rothman A. Evolution of the Biopsychosocial Model: Prospects and Challenges for HealthPsychology Health Psychology 2004;23(2):119-125. doi: 10.1037/0278-6133.23.2.119.
10. Ferrari CKB, Nery LD, Kopp MT, Santos DF, Perreira NS, Ferrari GSL, Barcelos FA, Gonçalves JS, Costa RJ. Saúde na escola: educação, saúde e inclusão em adolescentes brasileiros. Revista sobre la infancia y la adolescência 2013;4:78-90. doi: 10.4995/reinad.2013.1262.

11. Santos $A C$ de S. Prevalência de dor na coluna e sua relação com os hábitos posturais de escolares do ensino fundamental II na cidade de Campina Grande-PB. 2013. 23f. Trabalho de Conclusão de Curso (Graduação em Fisioterapia) Universidade Estadual da Paraíba.

12. Lemos AT, Santos FR dos, Moreira RB, Machado DT, Braga FCC, Gaya ACA. Ocorrência de dor lombar e fatores associados em crianças e adolescentes de uma escola privada do sul do Brasil. Cad Saúde Pública 2013;29(11):2177-2185. doi: 10.1590/0102-311x00030113.

13. Espandar R, Mortazavi SMJ, Baghdadi T. Angular Deformities of the Lower Limb in Children. Asian $\mathrm{J}$ Sports Med. 2010;1(1):46-53. doi: 10.5812/asjsm.34871.

14. Badaró, AFV, Nichele, LFI, Turra, P. Investigação da postura corporal de escolares em estudos brasileiros. Fisioterapia e Pesquisa 2015;22(2):197-204. doi: 10.590/18092950/13384622022015.

15. Braccialli LMP, Vilarta R. Aspectos a serem considerados na elaboração de programas de prevenção e orientação de problemas posturais. Rev Pau Educ Fís 2000;14(1):16-28

16. Meireles HR, Azevedo JH, Lopes Junior JEG, Figueiredo ADJ. Influência das Carteiras Escolares na Postura de Alunos da Rede Pública do Município de Cajazeiras-PB. Rev Fisioter Saúde Func 2013;2(1):35-41.

17. Macedo RB, Silva MJC, Sousa NF, Santos JV, Rodrigues AMM, Cumming SP, Lima AV, Gonçalves RS, Martins RA. Qualidade de vida, peso das mochilas escolares e lombalgia não específica em crianças e adolescentes. J Pediatr 2015;91(3):263-69. doi: 10.1016/j.jped.2014.08.011.

18. Healthy children. Backpack Safety [internet]. American Academy of Pediatrics; 21 Nov. 2015. [Acessado em 09 jul. 2016]. Disponível em: https://www.healthychildren.org/ English/safety-prevention/at-play/Pages/Backpack-Safety.aspx

Como citar: MANSOUR, Kamila Mohammad Kamal; ROVEDA, Patricia Oliveira. Presença da fisioterapia preventiva no contexto escolar: educação e prevenção em saúde. Cinergis, Santa Cruz do Sul, v. 17, out. 2016. ISSN 2177-4005. Disponível em: <https://online.unisc.br/seer/index.php/cinergis/article/view/8063>. Acesso em: 11 out. 2016. doi:http://dx.doi. org/10.17058/cinergis.v17i0.8063. 